\title{
Computationally efficient near-field source localization using third-order moments
}

\author{
Jian Chen*, Guohong Liu* and Xiaoying Sun
}

\begin{abstract}
In this paper, a third-order moment-based estimation of signal parameters via rotational invariance techniques (ESPRIT) algorithm is proposed for passive localization of near-field sources. By properly choosing sensor outputs of the symmetric uniform linear array, two special third-order moment matrices are constructed, in which the steering matrix is the function of electric angle $\gamma$, while the rotational factor is the function of electric angles $\gamma$ and $\phi$. With the singular value decomposition (SVD) operation, all direction-of-arrivals (DOAs) are estimated from a polynomial rooting version. After substituting the DOA information into the steering matrix, the rotational factor is determined via the total least squares (TLS) version, and the related range estimations are performed. Compared with the high-order ESPRIT method, the proposed algorithm requires a lower computational burden, and it avoids the parameter-match procedure. Computer simulations are carried out to demonstrate the performance of the proposed algorithm.
\end{abstract}

Keywords: Third-order moment; ESPRIT; Near-field source localization

\section{Introduction}

In the last decades, low-complexity parameter estimation has become an important topic required for radar, sonar, as well as communication [1,2]. Various efficient solutions have been developed to cope with this issue. However, most of these algorithms mainly focused on the far-field source localization, such as the multiple signal classification (MUSIC) method [3], estimation of signal parameters via rotational invariance techniques (ESPRIT) method [4], and their derivatives [5,6]. In order to locate the near-field sources [7], several effective methods have also been derived, which can be sorted as the following two versions. The first version is named as spectral searching methods, in which the two-dimensional (2-D) MUSIC [8] was the original solution to the nearfield source localization problem. To cope with the computationally inefficient 2-D searching procedure, a symmetric uniform linear array-based method [9] was proposed. Based on [9], resorting to the polynomial rooting, an improved method [10] was developed, which

*Correspondence: chenjian@jlu.edu.cn; liugh10@mails.jlu.edu.cn College of Communication Engineering, Jilin University, Changchun, Jilin 130025, China has further avoided the one-dimensional (1-D) searching procedure.

The other version, based on the rotational invariance of the underlying signal subspace induced by the translational invariance of the sensor array, is named as the closed-form solutions. In this version, the high-order ESPRIT method $[11,12]$ is especially noteworthy. This method was based on the cumulant domain data and adopted the total least squares (TLS) solution to obtain the direction-of-arrival (DOA) and range estimations, which avoided the computationally inefficient 2-D spectral search. However, two slight disadvantages of the high-order ESPRIT method are that (1) the construction of four cumulant matrices leads to an additional computational burden and (2) a parameter-pairing procedure is required for avoiding the estimation failure problem.

In this paper, we present a computationally more efficient ESPRIT algorithm to locate near-field sources. The main task in the first step is to estimate the electric angle $\gamma$, whereas in the second step, $\phi$ related to $\gamma$ is estimated for each near-field source. For the sake of reducing the computational load, we compute the thirdorder moments of sensor outputs and construct only two special $(N \times N)$-dimensional matrices $\mathbf{M}_{1}$ and $\mathbf{M}_{2}$.

\section{Springer}

(c) 2014 Chen et al.: licensee Springer. This is an Open Access article distributed under the terms of the Creative Commons Attribution License (http://creativecommons.org/licenses/by/4.0), which permits unrestricted use, distribution, and reproduction in any medium, provided the original work is properly credited. 
Since the steering matrix is the function of only electric angle $\gamma$, we can adopt the polynomial rooting solution to obtain the DOA estimations for all near-field sources. Instead of estimating two rotational factors from different eigenvalue-decompositions (EVDs) encountered in $[11,12]$, the proposed algorithm estimates the only one rotational factor from the TLS version, which can avoid the unknown permutation ambiguity accompanied by EVD, that is, the auto-pairing for DOA and range can be realized.

The remainder of this paper is organized as follows: Section 2 describes the near-field source localization model. In Section 3, an efficient third-order momentbased ESPRIT algorithm is proposed to deal with the nearfield source localization problem, and the performance analysis of the proposed algorithm is also addressed. Section 4 shows the simulation results. Section 5 presents the conclusion of the whole paper.

\section{Near-field signal model}

Suppose that $M$ near-field signals impinge on a symmetric uniform linear array (ULA) introduced by Liang and Yang [13]. This sensor array consists of $L=2 N+1$ sensors with element spacing $d$, and its array center is the phase reference point. With a proper rate that satisfies the Nyquist rate, the sampled signal received by the $l$ th sensor can be written as

$$
x_{l}(t)=\sum_{m=1}^{M} s_{m}(t) e^{j \tau_{l m}}+n_{l}(t),
$$

where $s_{m}(t)$ is the source signal, $n_{l}(t)$ is the additive sensor noise, and $\tau_{l m}$ is the delay associated with the $m$ th source signal propagation time from 0 th to $l$ th sensor. After being approximated by the Fresnel approximation, $\tau_{l m}$ has the following form $[11,12]$ :

$$
\tau_{l m}=l \gamma_{m}+l^{2} \phi_{m}
$$

where $\gamma_{m}$ and $\phi_{m}$ are called electric angles and given by

$$
\begin{aligned}
& \gamma_{m}=-2 \pi \frac{d}{\lambda} \sin \theta_{m} \\
& \phi_{m}=\pi \frac{d^{2}}{\lambda r_{m}} \cos ^{2} \theta_{m}
\end{aligned}
$$

where $\lambda$ represents the wavelength of the source signal and $\theta_{m}$ and $r_{m}$ denote azimuth DOA and range of the $m$ th near-field source signal, respectively. For the rest of this paper, the following assumptions are required to hold:

1) The source signals are statistically independent, zero-mean narrowband stationary processes with nonzero kurtosis;
2) The sensor noise is zero-mean Gaussian process, and independent of the source signals;

3) The sensor array is a symmetric ULA with element spacing $d \leq \lambda / 4$, and the source number $M$ is not more than the half of the sensor number $L$, which means $M<N$.

\section{The proposed algorithm}

In this section, we firstly review the definition of the third-order moment introduced in [14]. Then, by properly choosing the array outputs, two special third-order moment matrices are constructed, and a joint MUSICand ESPRIT-based solution is derived for the near-field source localization. Finally, the theoretical analysis including computational complexity and parameter match is carried out, which further evaluates the performance of the proposed algorithm.

\subsection{Construction of third-order moment matrix}

The proposed algorithm exploits the richness of the thirdorder moment of array outputs, which is defined as [14]

$$
\begin{aligned}
m_{3, x}(0, n,-n)= & \lim _{T \rightarrow \infty} \frac{1}{T} \sum_{t=1}^{T} \\
& \times E\left\{x_{0}(t) x_{n}\left(t+\tau_{1}\right) x_{-n}^{*}\left(t+\tau_{2}\right)\right\}
\end{aligned}
$$

where $n \in[-N, N]$, the superscript $*$ denotes conjugate operation, and $T$ represents the number of snapshots. Converging in the mean-square sense, the estimation of (5) is expressed as [14]

$$
m_{3, x}(0, n,-n)=\frac{1}{T} \sum_{t=1}^{T} x_{0}(t) x_{n}\left(t+\tau_{1}\right) x_{-n}^{*}\left(t+\tau_{2}\right) .
$$

With the given signal model (1), we further obtain

$$
\begin{aligned}
m_{3, x}(0, n,-n)= & \frac{1}{T} \sum_{t=1}^{T} \sum_{m=1}^{M} s_{m}(t) s_{m}\left(t+\tau_{1}\right) s_{m}^{*}\left(t+\tau_{2}\right) \\
& \times e^{j \tau_{n m}} e^{-j \tau_{-n m}} \\
= & \sum_{m=1}^{M} \frac{1}{T} \sum_{t=1}^{T} s_{m}(t) s_{m}\left(t+\tau_{1}\right) s_{m}^{*}\left(t+\tau_{2}\right) e^{j 2 n \gamma_{m}} \\
= & \sum_{m=1}^{M} m_{3, s_{m}}(\tau) e^{j 2 n \gamma_{m}}
\end{aligned}
$$

where $\tau=\tau_{1}+\tau_{2}$, and $m_{3, s_{m}}(\tau)$ represents the thirdorder moment of the $m$ th source signal. 
Based on (7), we can construct two spatial third-order moment matrices $\mathbf{M}_{1}$ and $\mathbf{M}_{2}$, in which the $(k, q)$ th elements are respectively given by

$$
\begin{aligned}
\mathbf{M}_{1}(k, q) & =m_{3, x}(0, k-q, q-k) \\
& =\sum_{m=1}^{M} m_{3, s_{m}}(\tau) e^{j 2(k-q) \gamma_{m}} \\
\mathbf{M}_{2}(k, q) & =m_{3, x}(-1, k-q, q-k) \\
& =\sum_{m=1}^{M} m_{3, s_{m}}(\tau) e^{j\left(-\gamma_{m}+\phi_{m}\right)} e^{j 2(k-q) \gamma_{m}} .
\end{aligned}
$$

In matrix form, we have

$$
\begin{aligned}
& \mathbf{M}_{1}=\mathbf{A M}_{3, s}(\tau) \mathbf{A}^{H} \\
& \mathbf{M}_{2}=\mathbf{A} \boldsymbol{\Omega} \boldsymbol{\Phi} \mathbf{M}_{3, s}(\tau) \mathbf{A}^{H}
\end{aligned}
$$

where the superscript $\mathrm{H}$ is conjugate transpose operation, $\mathbf{M}_{3, s}(\tau)$ is the third-order moment matrix of source signals, $\mathbf{A}=\left[\mathbf{a}_{1}, \mathbf{a}_{2}, \ldots, \mathbf{a}_{M}\right]$ is the $(N \times M)$-dimensional steering matrix, $\mathbf{a}_{m}=\left[1, e^{j 2 \gamma_{m}}, \ldots, e^{j 2(N-1) \gamma_{m}}\right]^{T}$ is the steering vector, and $\boldsymbol{\Omega} \boldsymbol{\Phi}$ is the $(M \times M)$-dimensional rotational factor and satisfies

$$
\begin{aligned}
& \boldsymbol{\Omega}=\operatorname{diag}\left(e^{-j \gamma_{1}}, e^{-j \gamma_{2}}, \ldots, e^{-j \gamma_{M}}\right) \\
& \boldsymbol{\Phi}=\operatorname{diag}\left(e^{j \phi_{1}}, e^{j \phi_{2}}, \ldots, e^{j \phi_{M}}\right) .
\end{aligned}
$$

Considering the third-order moment matrices in (10) and (11), one can form the $(2 N \times N)$-dimensional matrix

$$
\mathbf{M}=\left[\begin{array}{l}
\mathbf{M}_{1} \\
\mathbf{M}_{2}
\end{array}\right]=\left[\begin{array}{c}
\mathbf{A M}_{3, s}(\tau) \mathbf{A}^{H} \\
\mathbf{A} \boldsymbol{\Omega} \mathbf{\Phi} \mathbf{M}_{3, s}(\tau) \mathbf{A}^{H}
\end{array}\right] .
$$

\subsection{DOAs estimation for near-field sources}

In this paper, assume that the value of $M$ is known or correctly estimated by the Akaike information criterion (AIC) of the minimum description length (MDL) detection criterion [15]. Implementing a singular value decomposition (SVD) operation to $\mathbf{M}$ yields

$$
\mathbf{U T}=\left[\begin{array}{l}
\mathbf{U}_{1} \\
\mathbf{U}_{2}
\end{array}\right] \mathbf{T}=\left[\begin{array}{c}
\mathbf{A} \\
\mathbf{A} \boldsymbol{\Omega} \boldsymbol{\Phi}
\end{array}\right]
$$

where $\mathbf{U} \in C^{2 N \times M}$ is composed of the left singular vectors related to non-zero singular values, which spans the signal subspace of $\mathbf{M}, \mathbf{U}_{1} \in C^{N \times M}$, and $\mathbf{U}_{2} \in C^{N \times M}$ span the signal subspaces of $\mathbf{M}_{1}$ and $\mathbf{M}_{2}$, respectively, and the matrix $\mathbf{T} \in C^{M \times M}$ is the unique non-singular one.
Note that the azimuth DOAs for all the incoming sources can be estimated by finding the $M$ peaks from the following 1-D MUSIC spectral function

$$
P(\theta)=\left(\mathbf{a}(\theta)^{\mathbf{H}}\left(\mathbf{I}-\mathbf{U}_{1} \mathbf{U}_{1}^{\mathbf{H}}\right) \mathbf{a}(\theta)\right)^{-1}
$$

where I denotes the identity matrix.

In order to reduce the computational burden and improve the estimation accuracy, we obtain search-free estimator of DOAs based on polynomial rooting.

Denote $z=e^{j \gamma}$, we have

$$
\mathbf{a}(z)=\left[1, z, z^{2}, \cdots, z^{N}\right]^{T}
$$

The spectral function (16) can be rewritten in the following polynomial $[16,17]$

$$
P(z)=\mathbf{a}^{T}(1 / z)\left(\mathbf{I}-\mathbf{U}_{1} \mathbf{U}_{1}^{\mathbf{H}}\right) \mathbf{a}(z)
$$

and the DOAs of all the incoming sources can be estimated from the $M$ closest to the unit circle roots of (18).

\subsection{Range estimation for near-field sources}

With the obtained azimuth DOA information, we can easily obtain the estimations (denoted by $\tilde{\mathbf{A}}$ and $\overline{\boldsymbol{\Omega}}$ ) of $\mathbf{A}$ and $\boldsymbol{\Omega}$. Consider the total least squares (TLS) solution of the relation $\mathbf{U}_{1} \mathbf{T}=\mathbf{A}$, let $\mathbf{V}$ be the $(2 M \times 2 M)$-dimensional matrix of the right singular vectors of $\left[\begin{array}{ll}\mathbf{U}_{1} & \tilde{\mathbf{A}}\end{array}\right]$, if the matrix is divided into four $(M \times M)$-dimensional partitions as $[11,12]$

$$
\mathbf{V}=\left[\begin{array}{ll}
\mathbf{V}_{11} & \mathbf{V}_{12} \\
\mathbf{V}_{21} & \mathbf{V}_{22}
\end{array}\right]
$$

then the solution for the TLS problem is given by

$$
\tilde{\mathbf{T}}_{\mathrm{TLS}}=-\mathbf{V}_{12} \mathbf{V}_{22}^{-1}
$$

A similar approach will lead to the solution of $\boldsymbol{\Omega} \boldsymbol{\Phi}$

$$
(\tilde{\boldsymbol{\Omega}} \tilde{\boldsymbol{\Phi}})_{\mathrm{TLS}}=-\mathbf{E}_{12} \mathbf{E}_{22}^{-1}
$$

where $\mathbf{E}_{12}$ and $\mathbf{E}_{22}$ are the corresponding partitions of the matrix $\mathbf{E}$, which is generated from the right singular vectors of $\left[\begin{array}{lll}\tilde{\mathbf{A}} & \mathbf{U}_{2} & \tilde{\mathbf{T}}_{\mathrm{TLS}}\end{array}\right]$. 
Therefore, the estimation $\tilde{\phi}_{m}$ related to $\tilde{\gamma}_{m}$ can be determined as follows:

$$
\tilde{\phi}_{m}=\operatorname{angle}\left((\tilde{\boldsymbol{\Omega}} \tilde{\boldsymbol{\Phi}})_{\mathrm{TLS}}(m, m)\right)-\operatorname{angle}(\overline{\boldsymbol{\Omega}}(m, m)) .
$$

Finally, the range estimation of near-field sources is given by

$$
\tilde{r}_{m}=\pi \frac{d^{2}}{\lambda \tilde{\phi}_{m}} \cos ^{2} \tilde{\theta}_{m} .
$$

\subsection{Theoretical analysis of the proposed algorithm}

The proposed algorithm is based on a symmetric ULA with odd-numbered sensors, but the high-order ESPRIT method $[11,12]$ requires even ones. For the sake of simplicity, we assume that the two algorithms adopt an array of $2 N+2$ sensors and that of $2 N+1$ ones in the rest of this paper. In this section, we assess the performance of the proposed method from two ways, i.e, computational complexity and parameter match.
1) Computational complexity: Regarding the computational complexity, we compare the major multiplications involved in statistical matrix construction, EVD or SVD implementation, and MUSIC spectrum search. The high-order ESPRIT method constructs four $(\mathrm{N}+1) \times(\mathrm{N}+1)$ dimensional cumulant matrices, and performs the EVD of one $(3 \mathrm{~N}+3) \times(3 \mathrm{~N}+3)$-dimensional matrix, so the resulting multiplications are in order of

$$
O\left(36(N+1)^{2} T+4 / 3(3 N+3)^{3}\right)
$$

where $T$ is the snapshot number. The proposed algorithm constructs two $(N \times N)$-dimensional third-order moment matrices $\mathbf{M}_{1}$ and $\mathbf{M}_{2}$, implements the SVD of one $(2 N \times N)$-dimensional matrix $\mathbf{M}$, as well as executes once root-polynomial construction and solution for DOA estimation, the resulting multiplications are in order of

$$
O\left(2 N^{2} T+8 / 3 N^{3}+2 M N\right)
$$

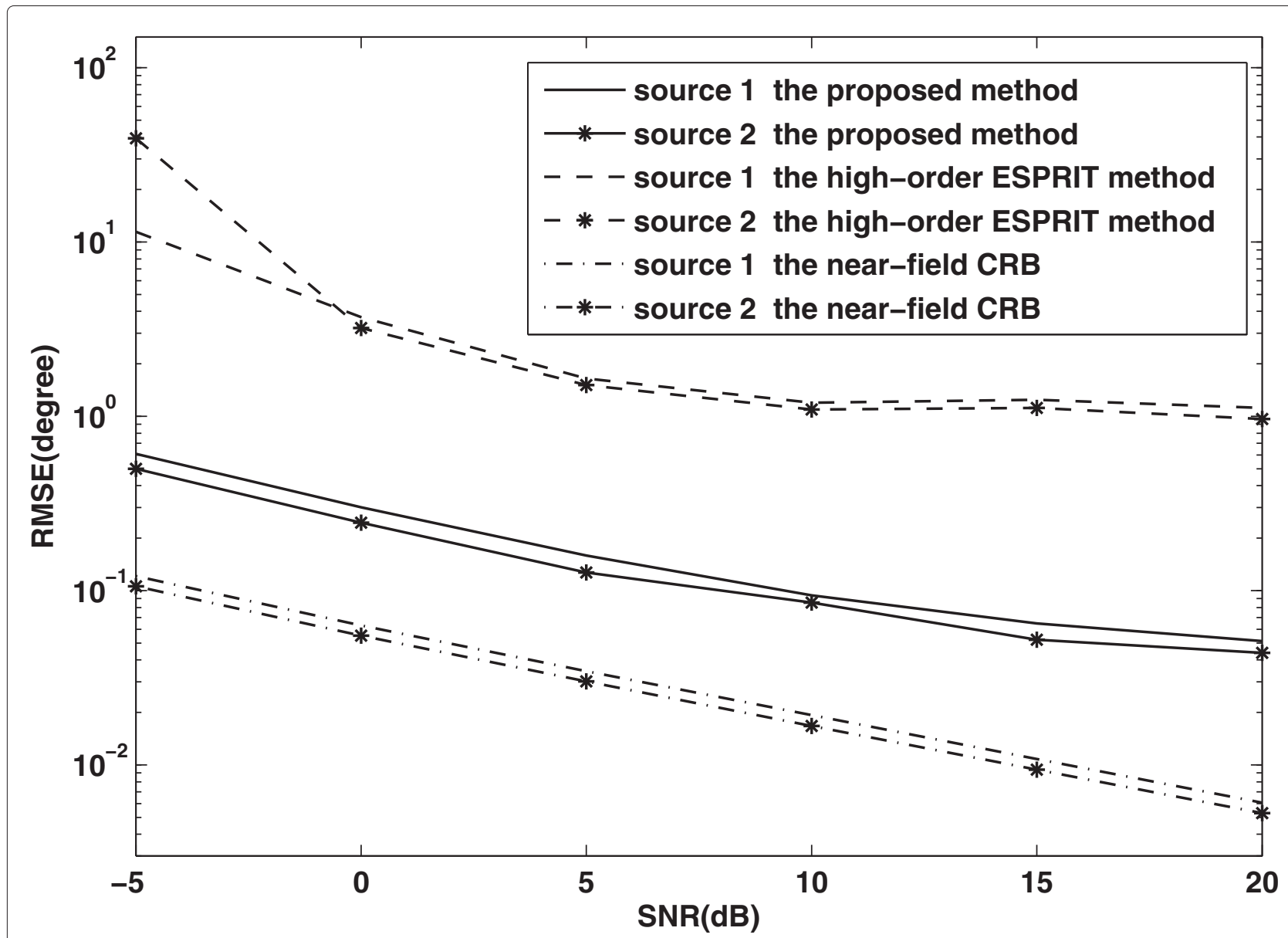

Figure 1 The RMSEs of azimuth DOA estimations for two near-field sources versus SNR. $\theta_{1}=35^{\circ}, r_{1}=0.3 \lambda, \theta_{2}=20^{\circ}, r_{2}=0.5 \lambda, T=1,024$, 500 independent trials. 
It is obvious that the proposed method is computationally more efficient than the high-order ESPRIT method.

2) Parameter match: The high-order ESPRIT method implements the EVDs of two matrices to separately estimate DOA and range of near-field sources. Although the eigenvectors related to non-zero eigenvalues from one EVD are equivalent to those of another EVD, the order may be different from each other. Therefore, an additional procedure is required to pairing them in a sense (see [7], section 2.4 for details). The proposed method firstly estimates DOAs using (16) and substitutes them into $\mathbf{A}$ and $\boldsymbol{\Omega}$ to obtain the estimations $\tilde{\mathbf{A}}$ and $\overline{\boldsymbol{\Omega}}$, respectively. Based on this, the estimation of $\boldsymbol{\Omega} \boldsymbol{\Phi}$ can be obtained from the TLS version shown in (19). Since there are no EVD operations for estimating the only one rotational factor $\boldsymbol{\Omega} \boldsymbol{\Phi}$, the unknown permutation ambiguity accompanied by EVD can be avoided, and the diagonal elements of $\tilde{\boldsymbol{\Omega}} \tilde{\boldsymbol{\Phi}}$ are in one-to-one correspondence with those of $\overline{\mathbf{\Omega}}$. That is, the auto-pairing for DOA and range is realized.

\section{Computer simulation results}

In this section, we explicit some simulation results to evaluate the performance of the proposed algorithm. For all examples, a symmetric ULA with 15 sensors and element spacing $0.25 \lambda$ is displayed. According to the definition in [13], the Fresnel region of the above array is $r \in(0.16 \lambda, 25 \lambda)$. The source signals are set as the zero-mean exponentially distributed ones. The sensor noise is assumed to be spatial white complex Gaussian, and the signal-to-noise ratio(SNR) is defined relative to each signal. For comparison, we simultaneously execute the high-order ESPRIT method [11,12] and the near-field Cramer-Rao bound (CRB) [18]. Note that the high-order ESPRIT method is based on a ULA with evennumbered sensors, so we adopt 16 sensors for it in the following experiments. The presented results are evaluated by the estimated root mean square error (RMSE)

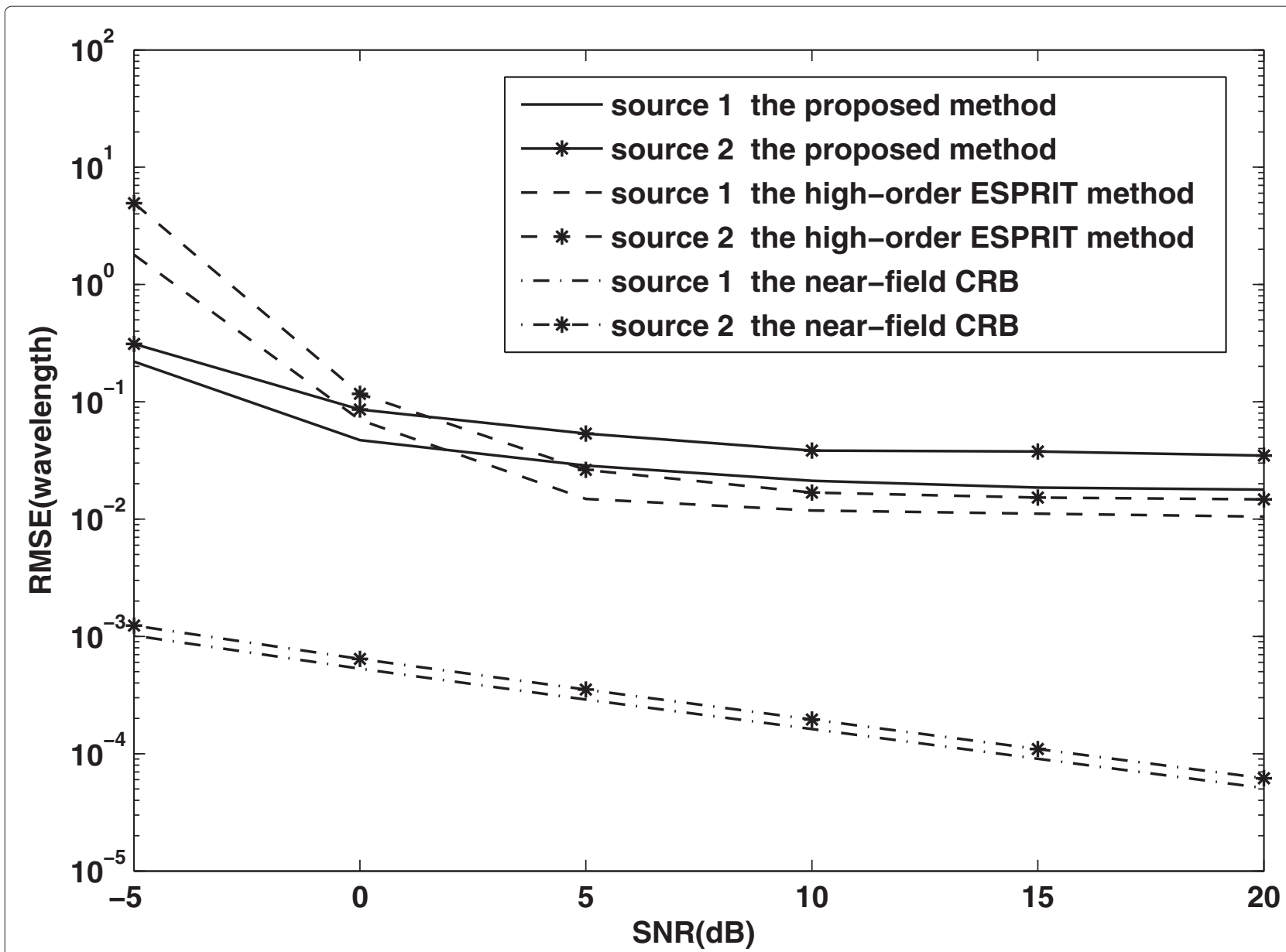

Figure 2 The RMSEs of azimuth range estimations for two near-field sources versus SNR. $\theta_{1}=35^{\circ}, r_{1}=0.3 \lambda, \theta_{2}=20^{\circ}, r_{2}=0.5 \lambda$, $T=1,024$, 500 independent trials. 
from the average results of 500 independent Monte Carlo simulations.

In the first example, we examine the estimation accuracy of the proposed algorithm versus the SNRs. The localization parameters for two near-field sources are $\left(35^{\circ}, 0.3 \lambda\right)$ and $\left(20^{\circ}, 0.5 \lambda\right)$, respectively. The thirdorder moment matrices are constructed using estimates $(T=1,024, \tau=0)$. When the SNR varies from -5 to $20 \mathrm{~dB}$, Figures 1 and 2 show the RMSEs of DOA and range estimations using the proposed algorithm, respectively.

For comparison, both the high-order ESPRIT method and the near-field CRB are also presented. It can be seen from Figure 1 that the proposed algorithm outperforms the high-order ESPRIT solution in estimating DOAs of two near-field sources, and the RMSEs are reasonably close to the near-field CRB. However, for the range estimation, the proposed algorithm slightly underperforms the high-order ESPRIT method. Note that the high-order ESPRIT method requires more computations than the proposed method, and it requires an additional parameter-pairing procedure.

In the second example, we assess the performance of the proposed algorithm versus the number of snapshots. The other simulation conditions are similar to the first example except that the SNR is set at $2 \mathrm{~dB}$, and the number of snapshots is varied from $T=400$ to $T=2,000$. The RMSEs of DOA and range estimations for the proposed method are displayed in Figure 3, and compared with the high-order ESPRIT method and the near-field CRB. We can see from this figure that the results are similar to those of the first example. The RMSEs of the proposed algorithm decrease monotonically with the number of snapshots. For the DOA estimations of both near-field signals, the proposed method shows a more satisfactory performance than the high-order ESPRIT method, and the related RMSEs are reasonably close to the near-field CRB.

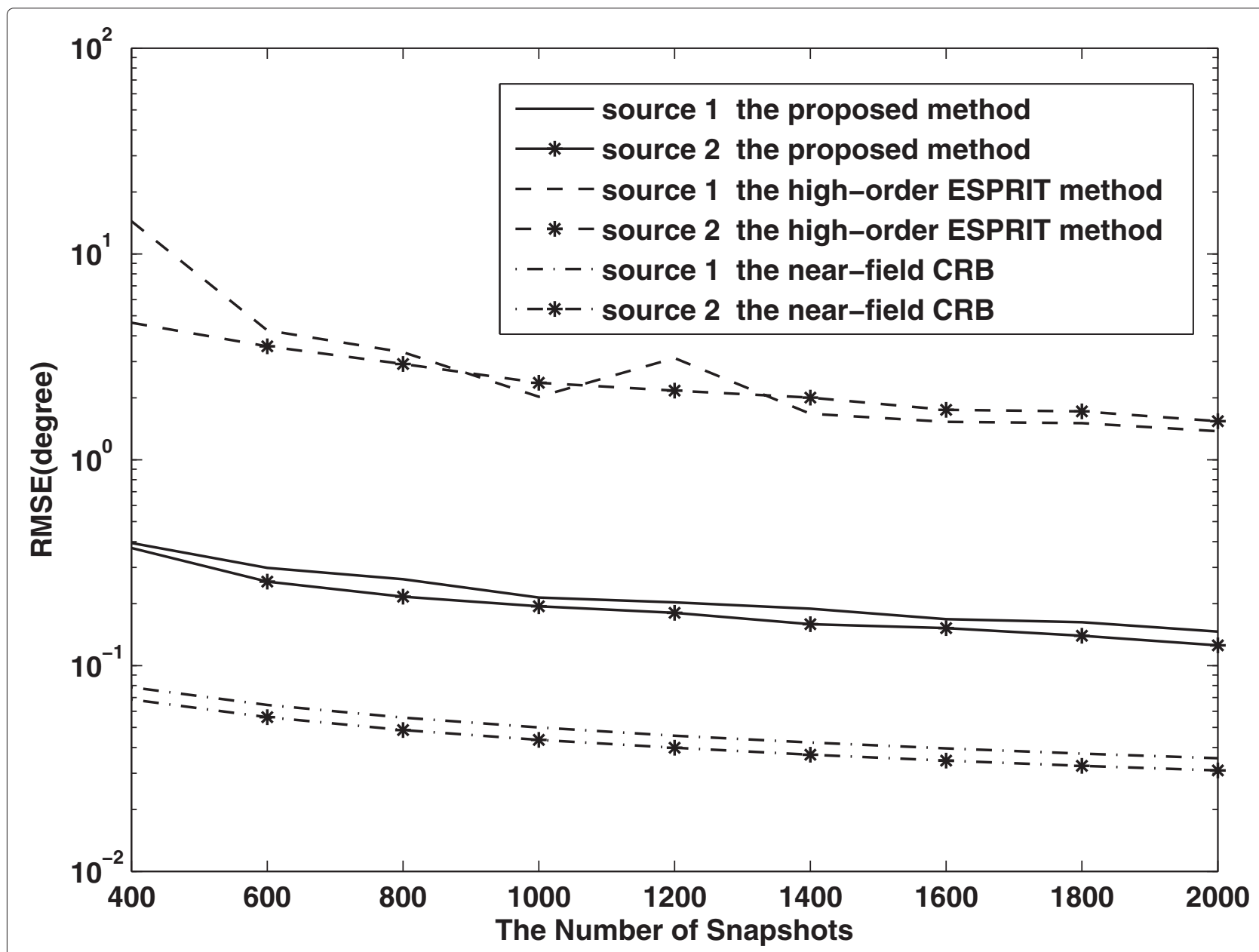

Figure 3 The RMSEs of azimuth DOAs estimations for two near-field sources versus the number of snapshots. $\theta_{1}=35^{\circ}, r_{1}=0.3 \lambda, \theta_{2}=20^{\circ}$, $r_{2}=0.5 \lambda, S N R=2 \mathrm{~dB}, 500$ independent trials. 


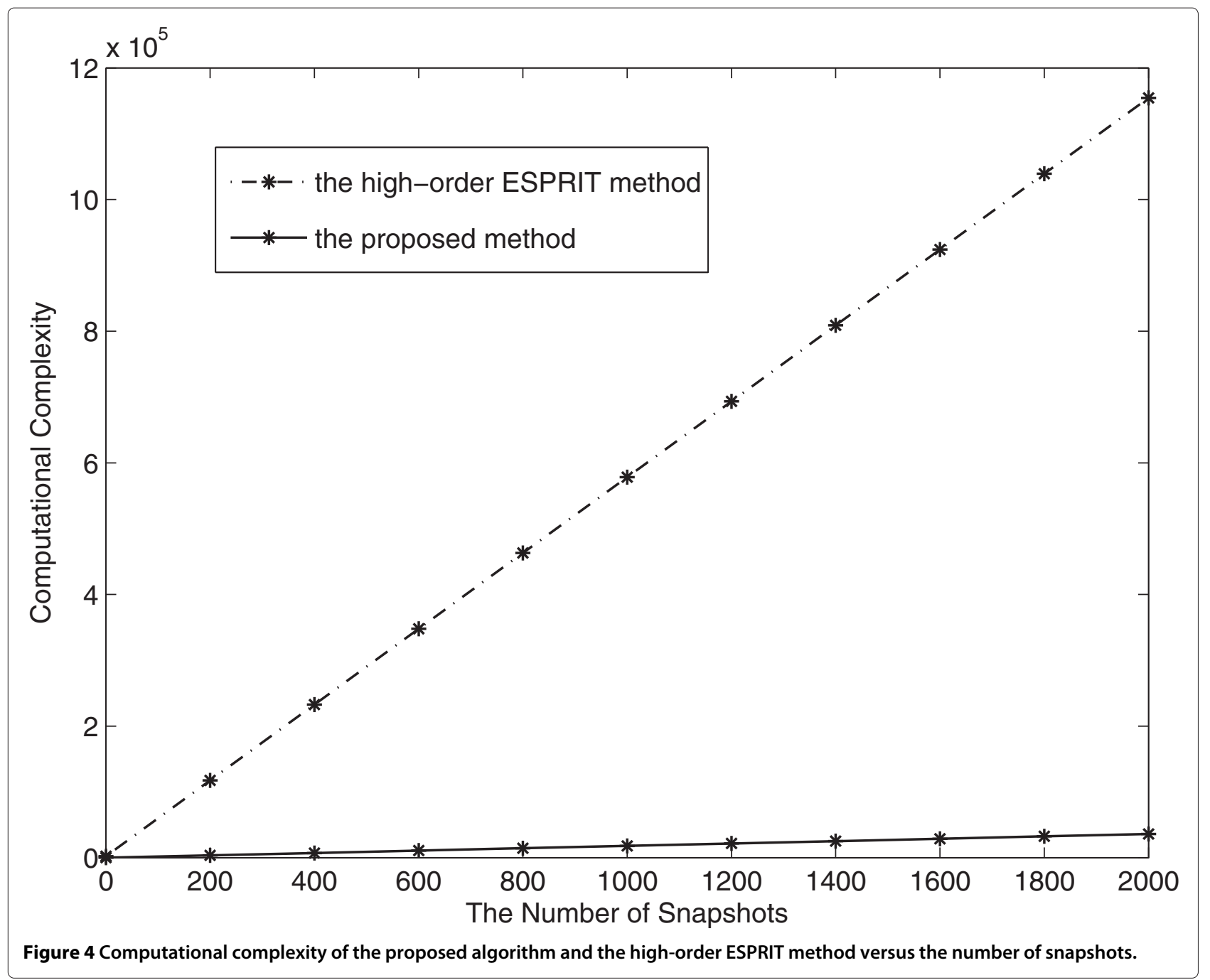

In the last example, the computational burden of the proposed algorithm is compared with the high-order ESPRIT method. The number of sensors is $2 N+2=10$. When the number of snapshots is varied from $T=0$ to $T=2,000$, Figure 4 shows the computational complexity of the proposed algorithm and that of the high-order ESPRIT method.

It can be seen from this figure that the proposed algorithm is computationally less complex than the high-order ESPRIT method.

\section{Conclusion}

This paper has presented a third-order moment-based ESPRIT method to cope with the near-field source localization problem. Our investigation has shown that the proposed method is capable of yielding reasonably good estimation of azimuth DOAs of near-field sources. Compared with the high-order ESPRIT method, the proposed method is efficient in the sense that it requires a lower computational burden, as well as realizes the auto-pairing for DOA and range.

\section{Competing interests}

The authors declare that they have no competing interests.

\section{Acknowledgements}

This work is supported by the National Nature Science Foundation of China (Grant 61171137) and 2009 New Century Excellent Talents in University (NCET) in China.

Received: 8 March 2014 Accepted: 14 June 2014

Published: 25 June 2014

\section{References}

1. C Qian, L Huang, Improved unitary root-MUSIC for DOA estimation based on pseudo-noise resampling. IEEE Signal Process. Lett. 21(2), 140-144 (2014)

2. F Liu, J Wang, C Sun, R Du, Spatial differencing method for DOA estimation under the coexistence of both uncorrelated and coherent signals. IEEE Trans. Antennas Propagation 60(4), 2052-2062 (2012)

3. RO Schmidt, Multiple emitter location and signal parameter estimation. IEEE Trans. Antennas Propagation 34(1), 276-280 (1986) 
4. R Rot, T Kailath, ESPRIT-estimation of signal parameters via rotational invariance techniques. IEEE Trans. Acoustics Speech Signal Process. 37(7), 984-995 (1989)

5. ML MeCloud, LL Scharf, A new subspace identification algorithm for high-resolution DOA estimation. IEEE Trans. Antennas Propagation 50(10), 1382-1390 (2002)

6. B Liao, SC Chan, Direction finding with partly calibrated uniform linear arrays. IEEE Trans. Antennas Propagation 60(2), 922-929 (2012)

7. SLi, BLF Daku, Optimal amplitude weighting for near-field passive source localization. IEEE Trans. Signal Process. 59(12), 6175-6185 (2011)

8. H Yung-Dar, M Barkat, Near-field multiple sources localization by passive sensor array. IEEE Trans. Antennas Propagation 39(7), 968-975 (1991)

9. W Zhi, MY-W Chia, Near-field source localization via symmetrix subarrays. IEEE Signal Process. Lett. 14(4), 409-412 (2007)

10. J-J Jiang, F-J Duan, J Chen, Y-C Li, Mixed far-field and near-field source localization using the uniform linear sensor array. IEEE Sensor J. 13(8), 3136-3143 (2013)

11. RN Challa, S Shamsunder, Higher order subspace based algorithm for passive localization of near-field sources, in The 1995 Conference Record of the Twenty-Ninth Asilomar Conference on Signals, Systems and Computers (Pacific Grove, CA, 30 Oct-2 Nov 1995), pp. 777-781

12. N Yuen, B Friedlander, Performance analysis of higher-order ESPRIT for localization of near-field sources. IEEE Trans. Signal Process. 46(3), 709-719 (1998)

13. J Liang, X Zeng, B Ji, J Zhang, F Zhao, A computationally efficient algorithm for joint range-doa-frequency estimation of near-field sources. Digit Signal Process. 19, 596-611 (2009)

14. AV Dandawate, GB Giannaks, Asymptotic theory of mixed time averages and kth-order cyclic-moment and cumulant statistics. IEEE Trans. Inf. Theory 41(1), 216-238 (1995)

15. M Wax, T Kailath, Detection of signals by information theoretic criteria. IEEE Trans. Signal Process. ASSP-33, 387-392 (1985)

16. BD Rao, KVS Hari, Performance analysis of root-MUSIC. IEEE Trans. Acoustic Speech Signal Process. 37(12), 1939-1949 (1989)

17. QS Ren, AJ Wills, Fast root MUSIC algorithm. Electron. Lett. 33(6), 450-451 (1997)

18. MNE Korso, R Boyer, A Renaux, S Marcos, Conditional and unconditional Cramér-Rao bounds for near-field source localization. IEEE Trans. Signal Process. 58(5), 2901-2907 (2010)

doi:10.1186/1687-6180-2014-98

Cite this article as: Chen et al.: Computationally efficient near-field source localization using third-order moments. EURASIP Journal on Advances in Signal Processing 2014 2014:98.

\section{Submit your manuscript to a SpringerOpen ${ }^{\mathcal{O}}$ journal and benefit from:}

- Convenient online submission

- Rigorous peer review

- Immediate publication on acceptance

- Open access: articles freely available online

- High visibility within the field

- Retaining the copyright to your article

Submit your next manuscript at $\boldsymbol{\wedge}$ springeropen.com 